British Journal of Nutrition (2023), 129, 115-125

doi:10.1017/S000711452200040X

(C) The Author(s), 2022. Published by Cambridge University Press on behalf of The Nutrition Society. This is an Open Access article, distributed under the terms of the Creative Commons Attribution licence (http://creativecommons.org/licenses/by/4.0/), which permits unrestricted re-use, distribution and reproduction, provided the original article is properly cited.

\title{
Association of alcohol types, coffee and tea intake with mortality: prospective cohort study of UK Biobank participants
}

\author{
Sylva M. Schaefer ${ }^{1}{ }^{*}$, Anna Kaiser ${ }^{1} \dagger$, Inken Behrendt ${ }^{1}$, Gerrit Eichner ${ }^{2} \ddagger$ and Mathias Fasshauer ${ }^{1}{ }^{3} \ddagger$ \\ ${ }^{1}$ Institute of Nutritional Science, Justus-Liebig University of Giessen, Giessen 35390, Germany \\ ${ }^{2}$ Mathematical Institute, Justus-Liebig University of Giessen, Giessen 35392, Germany \\ ${ }^{3}$ Department of Internal Medicine (Endocrinology, Nephrology, and Rheumatology), University of Leipzig, Leipzig 04103, \\ Germany
}

(Submitted 8 July 2021 - Final revision received 15 December 2021 - Accepted 31 January 2022 - First published online 3 February 2022)

\section{Abstract}

The present study examines how alcohol intake from wine and non-wine alcoholic beverages (non-wine) in $\mathrm{g} / \mathrm{d}$, as well as cups of coffee and tea included as continuous covariates and mutually adjusted are associated with all-cause, cancer, non-cancer and CVD mortality. Consumption was assessed in 354386 participants of the UK Biobank cohort who drank alcohol at least occasionally and survived at least 2 years after baseline with 20201 deaths occurring over 4.2 million person-years. Hazard ratios (HR) for mortality were assessed with Cox proportional hazard regression models and beverage intake fitted as penalised cubic splines. A significant U-shaped association was detected between wine consumption and all-cause, non-cancer and CVD mortality. Wine consumption with lowest risk of death (nadir) ranged from 19 to $23 \mathrm{~g}$ alcohol/d in all participants and both sexes separately. In contrast, non-wine intake was significantly and positively associated in a dose-dependent manner with all mortality types studied except for CVD in females and with the nadir between 0 and $12 \mathrm{~g}$ alcohol/d. In all participants, the nadir for all-cause mortality was 2 cups coffee/d with non-coffee drinkers showing a slightly increased risk of death. Tea consumption was significantly and negatively associated with all mortality types in both sexes. Taken together, light to moderate consumption of wine but not non-wine is associated with decreased allcause and non-cancer mortality. A minor negative association of coffee consumption with mortality cannot be excluded whereas tea intake is associated with a consistently decreased risk of all mortality types studied.

\section{Key words: Alcohol: Coffee: Metabolic syndrome: Mortality: Tea: UK Biobank: Wine}

Besides smoking and a sedentary lifestyle, unhealthy eating patterns are major contributors to morbidity, as well as all-cause and cause-specific mortality ${ }^{(1,2)}$. Various beverages including sugar- and artificially sweetened beverages have been implicated to contribute to metabolic and CVD, as well as cancer ${ }^{(3,4)}$. In contrast, for alcoholic drinks, coffee and tea, the association between intake and health effects is less clear.

Alcohol consumption is typically quantified in $\mathrm{g} / \mathrm{d}$ and the extent of alcohol intake is frequently divided into categories, including no, light, moderate and heavy drinking. Restricted alcohol intake $<16 \mathrm{~g} / \mathrm{d}$ is recommended for both sexes by the National Health Service to keep health risks low ${ }^{(5)}$. Chronic light to moderate drinking was associated with decreased risks of incident type 2 diabetes mellitus ${ }^{(6)}$, myocardial infarction and stroke ${ }^{(7)}$ but increased risks of hypertension in $\operatorname{men}^{(8)}$ and liver cirrhosis in women ${ }^{(9)}$. Moderate alcohol drinking was linked with an increased risk for oral/pharynx, oesophageal squamous cell, colorectal, liver, female breast cancer and malignant melanoma on one hand and a decreased risk for kidney, thyroid and haematologic malignancy on the other hand ${ }^{(10)}$. Based on these findings, light to moderate alcohol consumption is regarded as safe by the National Health Service; however, it is not recommended to start drinking alcohol or to drink more frequently to gain potential health benefits ${ }^{(5)}$. Several studies suggest that differences exist between wine and non-wine alcoholic beverages (non-wine) concerning their associations with incident diabetes mellitus ${ }^{(11)}$, obesity $^{(12)}$ and cancer ${ }^{(13)}$

Coffee intake is often quantified in cups/d with moderate coffee consumption defined as $3-5 \mathrm{cups} / \mathrm{d}^{(14)}$. Moderate coffee consumption was associated with decreased risks of incident

Abbreviations: AHI, annual household income; CI, confidence interval; HR, hazard ratio; MET, metabolic equivalent of task; OHR, overall health rating; PA, physical activity; Q, quartile.

* Corresponding author: S. M. Schaefer, fax +496419939069, email sylva.schaefer@ernaehrung.uni-giessen.de

$\dagger$ These authors contributed equally to this work as shared first authors

\$ These authors contributed equally to this work as shared senior authors. 
type 2 diabetes mellitus ${ }^{(15)}$, obesity ${ }^{(16)}, \mathrm{CVD}^{(17)}$, as well as liver and endometrial cancer ${ }^{(18)}$. Based on these results, moderate coffee consumption is considered safe ${ }^{(19)}$. Similar to coffee, moderate tea consumption is associated with decreased morbidity from type 2 diabetes mellitus ${ }^{(20)}$, ischaemic heart disease ${ }^{(21)}$, as well as several cancer subtypes ${ }^{(22)}$. Therefore, tea drinking is considered safe, but no specific guidelines exist concerning optimal intake.

Besides studies on the associations of alcohol, coffee and tea intake with morbidity, published evidence suggests that all-cause and cause-specific mortality are dose-dependently associated with the three beverages ${ }^{(23-30)}$. However, only few studies have assessed the association between wine $v$. non-wine consumption and mortality. Furthermore, models often include wine, non-wine, coffee and tea consumption as either linear predictors or even discretised ordinal predictors and mutual adjustments of the four beverages are not regularly performed. To address these limitations in the present study, associations between wine, non-wine, coffee and tea intake quantified on continuous scales and all-cause, as well as cause-specific, mortality hazards are determined in a large, well-characterised population of 354386 UK Biobank participants using penalised cubic splines to allow, in particular, non-linear predictor effects. We hypothesised that nonlinear relationships exist between the four beverage types and risk of death, as well as that intake levels linked to lowest mortality hazards depend on cause of death.

\section{Methods}

\section{Study and participants}

The study design of the multicentre, prospective UK Biobank cohort is described in detail at https://www.ukbiobank.ac. $\mathrm{uk}^{(31)}$. In brief, more than 500000 participants were recruited between 2006 and 2010 at twenty-two assessment centres across the UK with age at enrolment ranging from 38 to 73 years. At baseline, all participants were assessed by a self-completed touchscreen questionnaire, a personal interview and physical measurements as recently described ${ }^{(32)}$. The following five exclusion criteria (ec) were applied to all primary and sensitivity analyses: (1) missing smoking status; (2) missing socio-economic factors (i.e. ethnic background and/or overall health rating); (3) missing percentage body fat; (4) either missing information on beverage intake or being in the upper $0 \cdot 1 \%$ of alcohol, coffee or tea intake and (5) participants lost to follow-up or dying within 2 years after baseline (landmark analysis). All primary analyses with all outcome measures were performed in a primary cohort in which all non-alcohol drinkers were excluded in addition to ec1 to ec5 to remove all participants who might not drink alcohol due to health reasons (primary cohort; $n 354$ 386; online Supplementary Fig. 1). Non-alcohol drinkers were defined as participants with a present alcohol intake of $0 \mathrm{~g} / \mathrm{d}$. This group consisted of never drinkers, that is, participants never drinking alcohol during their lifetime, and former drinkers, that is, participants drinking alcohol in the past but not in the present. In addition to the primary analyses, two sets of sensitivity analyses (cohorts S1 and S2) were run. In the first set, former drinker bias was controlled for by excluding former drinkers but not never drinkers in addition to ec1-ec5 (cohort S1; $n 374$ 697). Since former drinkers include ex-drinkers who quit alcohol due to poor health, former drinker bias contributes to an apparently lower mortality of moderate drinkers $^{(33,34)}$. In the second set of sensitivity analyses, only ec1ec5 were applied, that is, non-drinkers were not excluded (cohort S2; $n 399$ 866). Therefore, this set of analyses did not control for health issues in participants not drinking alcohol. The UK Biobank study was approved by the North West Multicentre Research Ethics Committee and all participants provided written informed consent before inclusion ${ }^{(31)}$.

\section{Exposure assessment}

Estimation of alcohol from wine and non-wine, as well as coffee, and tea intake was performed similar as described by Bradbury and co-workers ${ }^{(35)}$. In brief, participants were asked about their weekly or monthly consumption of different alcoholic drinks during the baseline visit. Consumption of red wine and champagne plus white wine was included in the present analysis as wine intake whereas all other categories of alcoholic drinks, that is, beer plus cider, spirits, fortified wine and other alcoholic drinks, were included as non-wine. All alcoholic drinks were assumed to contain $10 \mathrm{~g}$ alcohol per portion except a pint of beer which was supposed to contain $20 \mathrm{~g}$ alcohol. Total weekly and monthly consumption of wine and non-wine was summed up for each participant. For an estimation of wine and non-wine intake in $\mathrm{g} / \mathrm{d}$, weekly and monthly consumption was divided by 7 and 30.4375, respectively. Furthermore, participants documented coffee and tea consumption as cups/d. An amount of 0.5 cups/d was assumed if 'less than one' cup of coffee or tea was recorded. Participants who did not indicate the extent of alcohol, coffee or tea intake including those answering 'do not know' or 'prefer not to answer' were excluded from the present analysis. In a subcohort of UK Biobank participants, only 'consumption of other alcoholic drinks' was not available. In these cases, this category was set to $0 \mathrm{~g}$ similar to Bradbury and coworkers $^{(35)}$.

\section{Outcome assessment}

Mortality data with date and underlying primary cause of death were provided by the National Health Service Information Centre for participants from England and Wales and by the National Health Service Central Register, Scotland for participants from Scotland ${ }^{(36)}$. Follow-up time was calculated between the date of baseline assessment and date of death or censoring (i.e. 23 March 2021), whichever came first. Besides overall mortality, the following two main mortality categories were defined according to the International Statistical Classification of Diseases and Related Health Problems, Tenth Revision (ICD-10) codes: cancer (C00-D48) and non-cancer (all but C00-D48). Furthermore, CVD (I00-I79) mortality was analysed. All analyses were performed in all participants, as well as in females and males separately. 
(a)

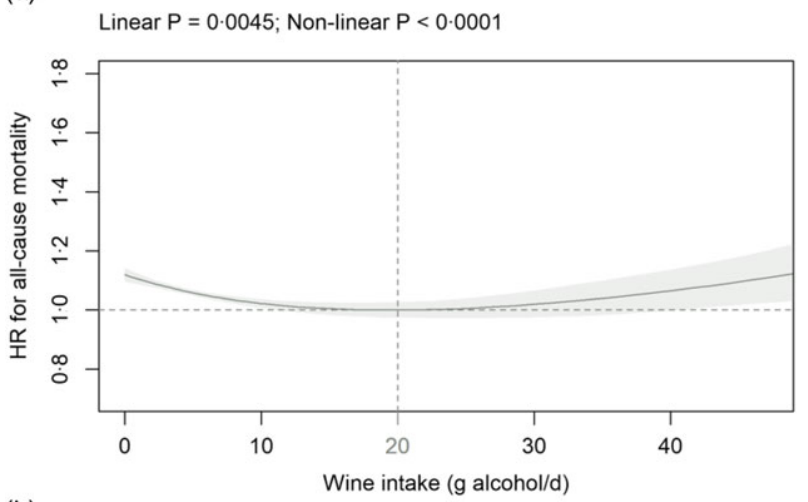

(b)

Linear $\mathrm{P}=0.0963 ;$ Non-linear $\mathrm{P}=0.0202$

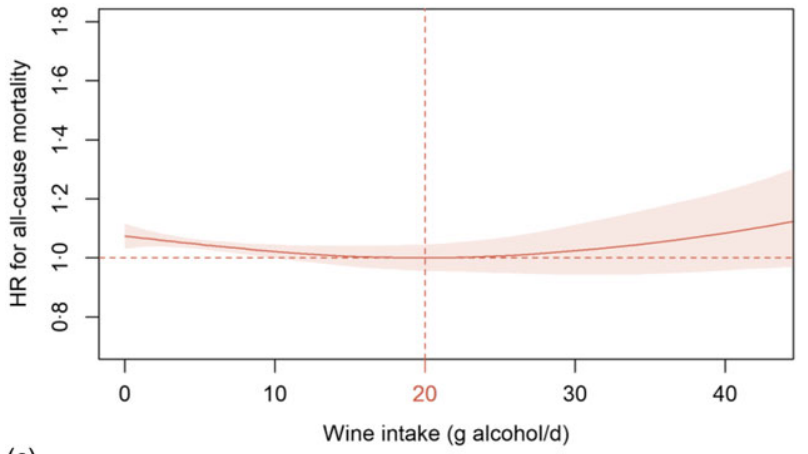

(c)

Linear $\mathrm{P}=0.0286$; Non-linear $\mathrm{P}<0.0001$

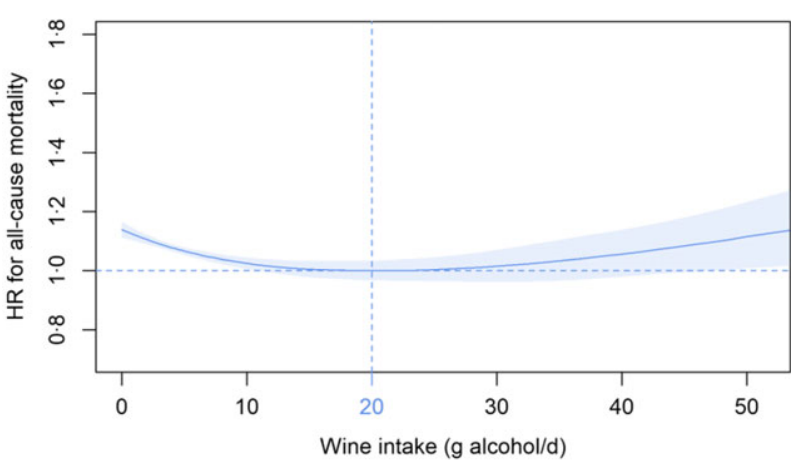

Fig. 1. Association of wine intake (g alcohol/d) in: (a) all participants; (b) females and (c) males with all-cause mortality in the primary cohort. Data are adjusted for sex (all participants only), age, AHI, ethnicity, OHR, PA, percentage body fat and smoking status. Additionally, wine, non-wine, coffee and tea intake are mutually adjusted (e.g. wine intake is additionally adjusted for non-wine, coffee and tea intake) as summarised in the Methods section. Covariates not fulfilling the proportional hazard assumption (all participants: age; females: age; males: age, $\mathrm{OHR}$, percentage body fat) are stratified. The nadir is indicated in grey (total cohort), red (female) and blue (male). HR: hazard ratio; AHI, annual household income; OHR, overall health rating; PA, physical activity.

\section{Statistical analyses}

Data were imported, processed, analysed and graphically displayed with $R$ version $4.0 .5^{(37)}$ in combination with the packages readxl $^{(38)}$ tidyverse $^{(39)}$, venn ${ }^{(40)}$, skimr ${ }^{(41)}$ and survival ${ }^{(42)}$. Cox proportional hazard regression models were fitted with wine, nonwine, coffee and tea mutually adjusted and included as penalised cubic splines with their degrees of freedom set to 4 . The analysis of each penalised cubic spline is segregated into its linear and (a)

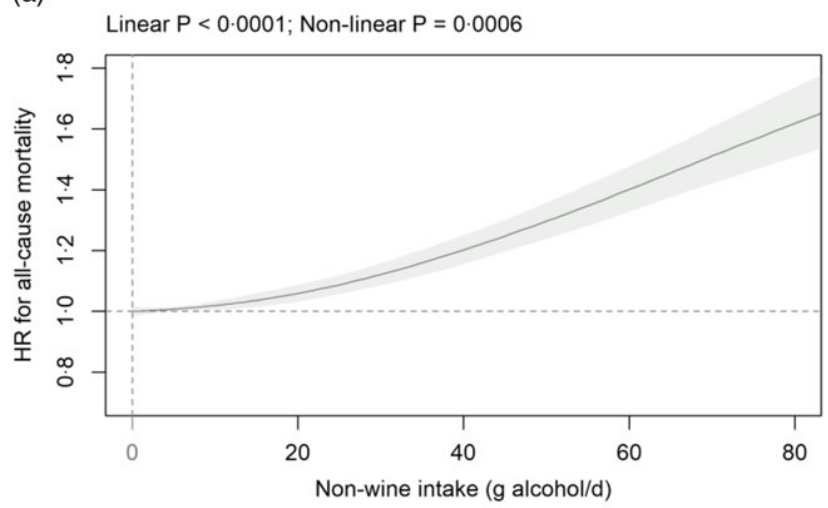

(b)

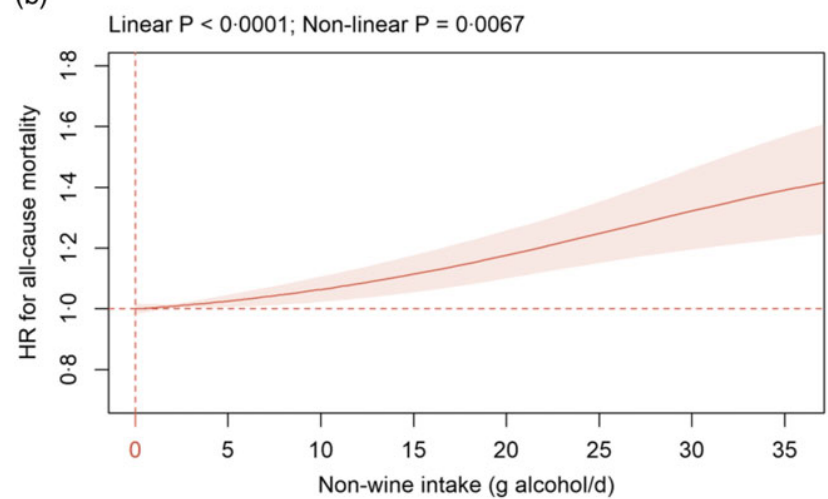

(c)

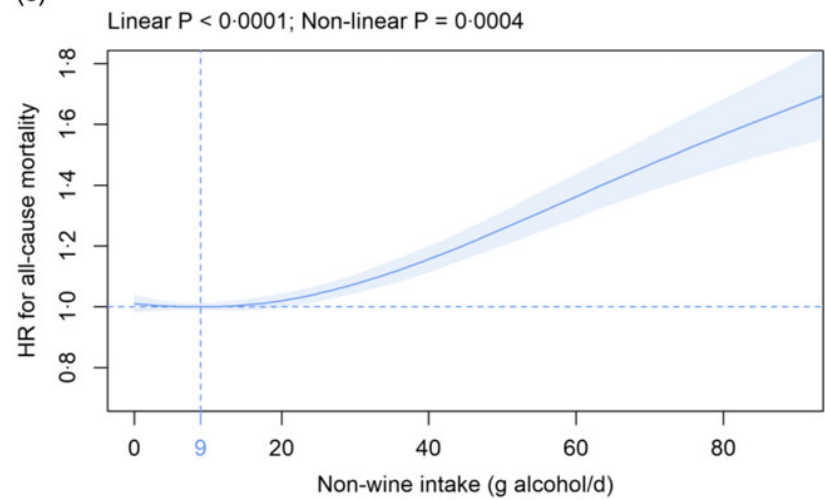

Fig. 2. Association of non-wine intake (g alcohol/d) in: (a) all participants; (b) females and (c) males with all-cause mortality in the primary cohort. Data are adjusted and presented as indicated in Fig. 1.

non-linear effects whose significances are documented by the respective $P$ values ( $\mathrm{p}^{\text {lin }}$ for the linear and $\mathrm{p}^{\text {non-lin }}$ for the non-linear effect) of Wald-type tests for joint significance of the multiple coefficients associated with the respective linear or non-linear portion of the penalised spline fit ${ }^{(43,44)}$. These $P$ values for the association of wine, non-wine, coffee and tea with mortality are depicted in Fig. 1-4, online Supplementary Fig. 2 and 3 and Supplementary Tables 2-4. In all analyses, the nadir was defined as the consumption of wine, non-wine, coffee and tea with the lowest estimated hazard ratio (HR) over the range from 0 to the $99 \%$ quantile of consumption and the HR at the nadir was set to 1 to simplify presentations and comparisons. HR with pointwise $95 \% \mathrm{CI}$ are shown for all mortality analyses. In all analyses, $\mathrm{HR}^{0}$ reflects $\mathrm{HR}$ in non- 
(a)

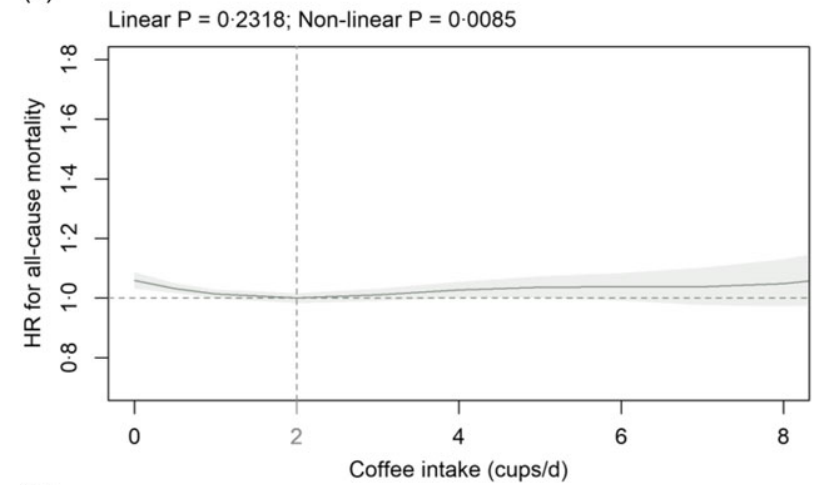

(b)

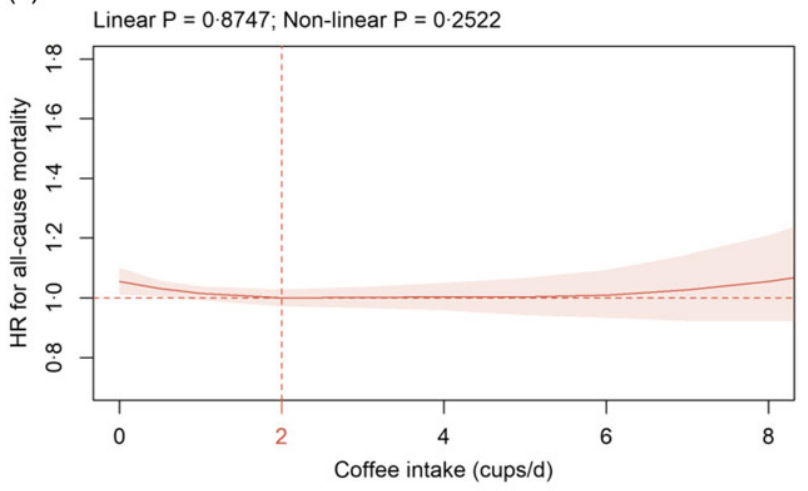

(c)

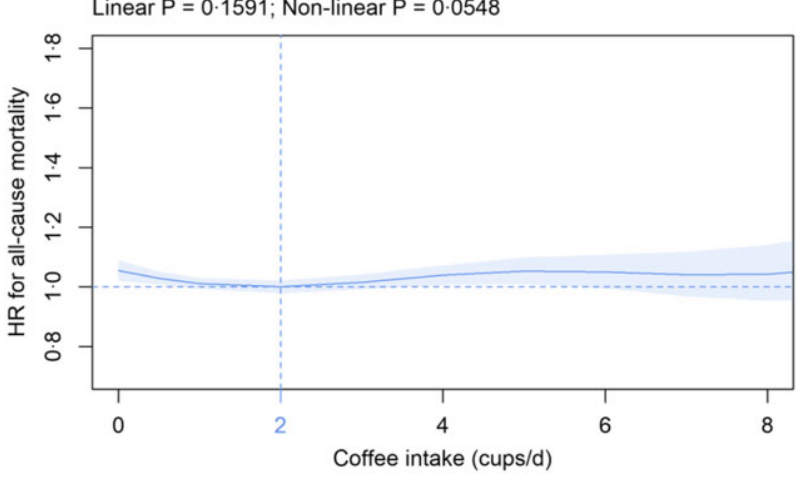

Fig. 3. Association of coffee intake (cups/d) in: (a) all participants; (b) females and (c) males with all-cause mortality in the primary cohort. Data are adjusted and presented as indicated in Fig. 1.

consumers of wine, non-wine, coffee or tea relative to the HR at the nadir. If both $\mathrm{p}^{\text {lin }}$ and $\mathrm{p}^{\text {non-lin }}$ were non-significant, no further interpretation of $\mathrm{HR}^{0}$, other individual $\mathrm{HR}$ or of the nadir was performed. For cause-specific mortality, survival times of participants with other causes of death were considered censored at their date of death. The proportional hazard assumption was tested based on scaled Schoenfeld residuals and all covariates violating this assumption after Holm adjustment for multiple testing were stratified in the final models and are defined in the figure and table legends. Models were adjusted for age (quartiles), sex (all participants only), total physical activity (PA; metabolic equivalent of task-min/week: $<1000,1000$ to $<2000,2000$ to $<4000, \geq 4000$ and unknown), smoking status (never, previous and current), annual household income (AHI; $<18,18$ to $<31,31$ to $<52,52$ to $<100, \geq 100 \mathrm{k£}$ and unknown), ethnicity (White, Group combined of Mixed, Asian, (a)

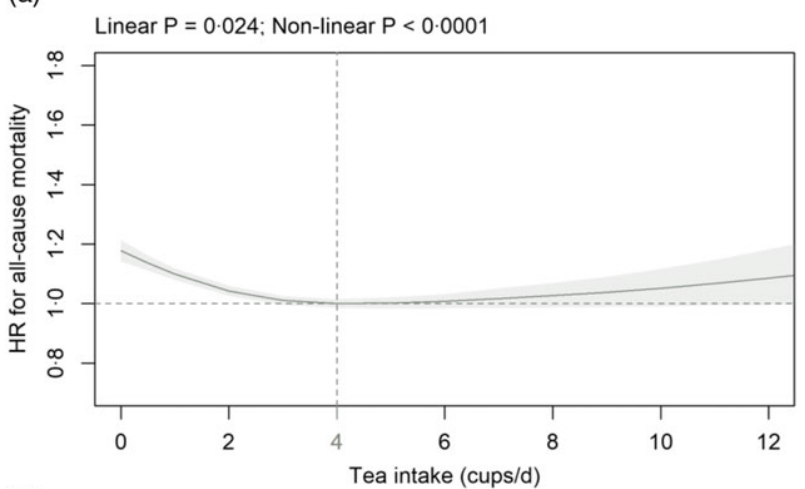

(b)

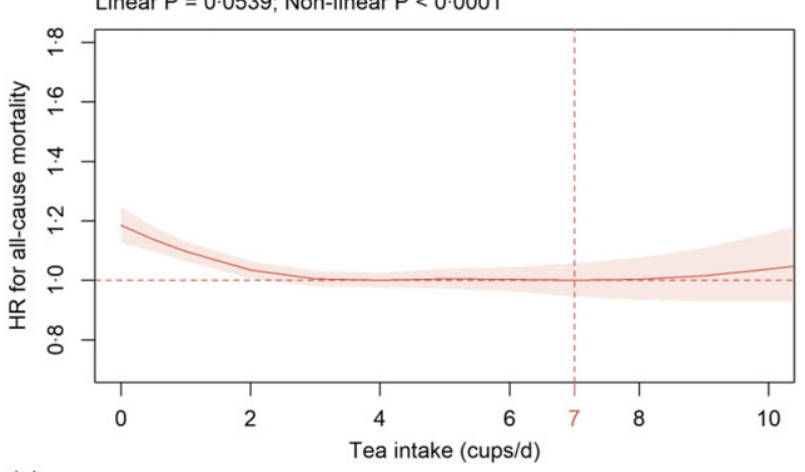

(c)

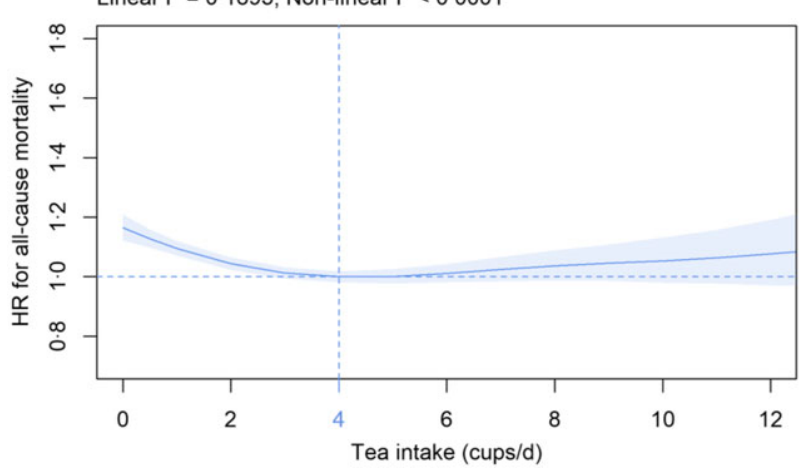

Fig. 4. Association of tea intake (cups/d) in: (a) all participants; (b) females and (c) males with all-cause mortality in the primary cohort. Data are adjusted and presented as indicated in Fig. 1.

Black, Chinese and Other) and overall health rating (OHR; poor, fair, good and excellent). Since percentage body fat is also an independent and significant predictor of all-cause mortality (data not shown), all analyses were adjusted for this covariate (quartiles) as well. A $P$ value of $<0.05$ was considered as statistically significant in all analyses.

\section{Results}

\section{Baseline characteristics and deaths in UK Biobank participants}

Baseline characteristics of the study population in total and depending on wine, non-wine, coffee and tea intake are summarised in Table 1. Median (Q (Quartile) 1, Q3) age of the study 
NS British Journal of Nutrition

Table 1. Baseline characteristics of the UK Biobank cohort* (median values and quartiles)

\begin{tabular}{|c|c|c|c|c|c|c|c|c|c|c|c|c|c|c|c|c|c|c|c|c|c|}
\hline \multirow[b]{3}{*}{ Parameter } & \multirow{2}{*}{\multicolumn{4}{|c|}{$\begin{array}{c}\text { Total cohort } \\
n 354386 \\
\end{array}$}} & \multicolumn{8}{|c|}{ Wine intake in $\mathrm{g}$ alcohol/d } & \multicolumn{9}{|c|}{ Non-wine intake in $\mathrm{g}$ alcohol/d } \\
\hline & & & & & \multicolumn{2}{|c|}{$<1 n 74357$} & \multicolumn{2}{|c|}{$1-<8 \cap 127737$} & \multicolumn{2}{|c|}{$8-<16 n 89845$} & \multicolumn{2}{|c|}{$\geq 16 n 62447$} & \multicolumn{3}{|c|}{$<1$ n 104546} & \multicolumn{2}{|c|}{$1-<8 \cap 118149$} & \multicolumn{2}{|c|}{$8-<16$ n 58126} & \multicolumn{2}{|c|}{$\geq 16 n 73565$} \\
\hline & \multicolumn{2}{|c|}{ Median } & \multicolumn{2}{|c|}{ Q1, Q3 } & Median & Q1, Q3 & Median & Q1, Q3 & Median & Q1, Q3 & Median & Q1, Q3 & Median & & , Q3 & Median & Q1, Q3 & Median & Q1, Q3 & Median & Q1, Q3 \\
\hline $\begin{array}{l}\text { Wine intake (g alco- } \\
\text { hol/d) }\end{array}$ & & 5.7 & 1.4, & 11.4 & 0.0 & $0.0,0.0$ & $4 \cdot 3$ & $2 \cdot 9,5 \cdot 7$ & $10 \cdot 0$ & $8.6,12 \cdot 9$ & $21 \cdot 4$ & $17 \cdot 1,30.0$ & & $2 \cdot 9$ & , $14 \cdot 3$ & 5.7 & $2 \cdot 9,11 \cdot 4$ & $5 \cdot 7$ & $1 \cdot 4,12 \cdot 9$ & $2 \cdot 9$ & $0 \cdot 0,10 \cdot 0$ \\
\hline $\begin{array}{l}\text { Non-wine intake (g } \\
\text { alcohol/d) }\end{array}$ & & $4 \cdot 3$ & 0.0 & $12 \cdot 9$ & $10 \cdot 0$ & $2 \cdot 6,25 \cdot 7$ & $2 \cdot 9$ & $0.0,8 \cdot 6$ & $2 \cdot 9$ & $0.0,10.0$ & $4 \cdot 3$ & $0.0,11 \cdot 4$ & & 0.0 & 0.0 & $2 \cdot 9$ & $2 \cdot 9,5 \cdot 7$ & 11.4 & $8 \cdot 6,12 \cdot 9$ & 28.6 & $20 \cdot 0,42 \cdot 9$ \\
\hline Coffee intake (cups/d) & & 2.0 & 0.5, & 3.0 & $1 \cdot 0$ & $0.0,3.0$ & 2.0 & $0.5,3.0$ & 2.0 & $1 \cdot 0,3 \cdot 0$ & $2 \cdot 0$ & $1 \cdot 0,3 \cdot 0$ & & 0.5 & 3.0 & 2.0 & $1 \cdot 0,3 \cdot 0$ & $2 \cdot 0$ & $1 \cdot 0,3 \cdot 0$ & $2 \cdot 0$ & $0.5,3.0$ \\
\hline Tea intake (cups/d) & & $\begin{array}{r}3.0 \\
50\end{array}$ & $1 \cdot 0,5$ & & 3.0 & $1.0,5 \cdot 0$ & $\begin{array}{r}3.0 \\
50\end{array}$ & $2 \cdot 0,5 \cdot 0$ & 3.0 & $1.0,5.0$ & 3.0 & $1 \cdot 0,5.0$ & & $1 \cdot 0$ & 5.0 & $\begin{array}{r}3.0 \\
50\end{array}$ & $2.0,5 \cdot 0$ & 3.0 & $1.0,5.0$ & 3.0 & $1 \cdot 0,5 \cdot 0$ \\
\hline Age (years) & & 58 & 50, & & 57 & 49,63 & 58 & 50,63 & 58 & 50,63 & 57 & 50,63 & 58 & & 63 & 58 & 50,63 & 57 & 50,63 & 57 & 50,63 \\
\hline $\begin{array}{l}\text { Female } \\
\text { Smoking status }\end{array}$ & 179 & $\begin{array}{c}n \\
676\end{array}$ & $\begin{array}{l}\% \\
50.7\end{array}$ & & $\begin{array}{r}n \\
28548\end{array}$ & 8.4 & $\begin{array}{r}n \\
71264\end{array}$ & $\begin{array}{l}\% \\
55.8\end{array}$ & $\begin{array}{c}n \\
50053\end{array}$ & $\begin{array}{l}\% \\
55.7\end{array}$ & $\begin{array}{r}n \\
29811\end{array}$ & $\begin{array}{l}\% \\
47.7\end{array}$ & $\begin{array}{r}n \\
84040\end{array}$ & $\begin{array}{l}\% \\
80.4\end{array}$ & & $\begin{array}{r}n \\
68381\end{array}$ & $\%$ & $\begin{array}{r}n \\
17965\end{array}$ & $\begin{array}{l}\% \\
\% 0.9\end{array}$ & $\begin{array}{r}n \\
9290\end{array}$ & $\begin{array}{l}\% \\
12.6\end{array}$ \\
\hline Never & 1873 & 367 & 52.9 & & 6312 & 8.8 & 78243 & $61 \cdot 3$ & 47836 & $53 \cdot 2$ & 24976 & $40 \cdot 0$ & 61491 & 58.8 & & 70297 & 9.5 & 28390 & 48.8 & 27189 & 37.0 \\
\hline Previous & 1318 & 857 & $37 \cdot 2$ & & 6081 & 5.1 & 40786 & 31.9 & 35371 & 39.4 & 29619 & $47 \cdot 4$ & 36057 & 34.5 & & 39699 & 3.6 & 23414 & 40.3 & 32687 & 44.4 \\
\hline $\begin{array}{l}\text { Current } \\
\text { CHI }\end{array}$ & 351 & 162 & 9.9 & & 1964 & 6.1 & 8708 & 6.8 & 6638 & $7 \cdot 4$ & 7852 & $12 \cdot 6$ & 6998 & 6.7 & & 8153 & 6.9 & 6322 & $10 \cdot 9$ & 13689 & 18.6 \\
\hline 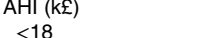 & & 502 & 15.9 & & 9987 & 6.9 & 19460 & 15.2 & 10510 & 11.7 & 6545 & 10.5 & 15118 & 14.5 & & 16891 & & & & & \\
\hline $18-<31$ & 769 & 979 & 21.7 & & 8087 & 4.3 & 28599 & 22.4 & 18451 & 20.5 & $\begin{array}{l}0540 \\
11842\end{array}$ & 19.0 & 22113 & 21.2 & & $\begin{array}{l}16891 \\
25146\end{array}$ & $4 \cdot 3$ & 9167 & $15 \cdot 8$ & 15326 & $20 \cdot 8$ \\
\hline $31-<52$ & 854 & 496 & 24.1 & & 5701 & 1.1 & 31366 & 24.6 & 22630 & 25.2 & 15799 & $25 \cdot 3$ & 24602 & 23.5 & & $\begin{array}{ll}25140 \\
28467\end{array}$ & 1.3 & 12594 & 27.7 & 17126 & $23 \cdot 3$ \\
\hline $52-<100$ & 719 & 907 & 20.3 & & 8546 & 1.5 & 25486 & 20.0 & 21416 & 23.8 & 16459 & 26.4 & 21598 & 20.7 & & 28467 & 4.7 & $\begin{array}{l}14 \\
1293 \\
\end{array}$ & 24.9 & 17934 & $24 \cdot 4$ \\
\hline$\geq 100$ & 201 & & 5.7 & & 1209 & 1.6 & 6076 & 4.8 & 6572 & & & $\begin{array}{l}10.1 \\
0.1\end{array}$ & & 6.6 & & & $1 \cdot 4$ & 12221 & $27 \cdot 0$ & 12831 & $17: 4$ \\
\hline & & & & & & & & & & & & & 0514 & & & 7483 & $6 \cdot 3$ & 3200 & 5.5 & 2575 & 3.5 \\
\hline Ethnicity & 433 & 330 & $12 \cdot 2$ & & 0872 & $4 \cdot 6$ & 16750 & $13 \cdot 1$ & 10266 & 11.4 & 5487 & 8.8 & 14201 & $13 \cdot 6$ & & 14911 & $2 \cdot 6$ & 6445 & $11 \cdot 1$ & 7773 & $10 \cdot 6$ \\
\hline White & 3426 & 689 & 96.7 & & 9987 & 4.1 & 122976 & $96 \cdot 3$ & 88179 & $98 \cdot 1$ & 61547 & $98 \cdot 6$ & 100224 & 95.9 & & 14063 & 6.5 & 56585 & 97.3 & 71817 & 97.6 \\
\hline $\begin{array}{l}\text { Mixed, Asian, Black, } \\
\text { Chinese, Other }\end{array}$ & 116 & 697 & $3 \cdot 3$ & & 4370 & 5.9 & 4761 & 3.7 & 1666 & 1.9 & 900 & 1.4 & 4322 & $4 \cdot 1$ & & 4086 & 3.5 & 1541 & 2.7 & 1748 & $2 \cdot 4$ \\
\hline & & & & & & & & & & & & & & & & & & & & & \\
\hline Excellent & 637 & 715 & 18.0 & & 8329 & 1.2 & 23702 & 18.6 & 19005 & $21 \cdot 2$ & 12679 & $20 \cdot 3$ & 21089 & $20 \cdot 2$ & & 22665 & 9.2 & 10215 & 17.6 & 9746 & 13.2 \\
\hline Good & 2129 & 997 & 60.1 & & 1093 & 5.3 & 78408 & 61.4 & 55666 & 62.0 & 37830 & 60.6 & 63880 & 61.1 & & 72421 & 1.3 & 34888 & 60.0 & 41808 & 56.8 \\
\hline Fair & 669 & 994 & $18 \cdot 9$ & & 0298 & 7.3 & 22620 & $17 \cdot 7$ & 13540 & $15 \cdot 1$ & 10536 & 16.9 & 17043 & $16 \cdot 3$ & & 20161 & $7 \cdot 1$ & 11342 & 19.5 & 18448 & $25 \cdot 1$ \\
\hline Poor & 106 & 680 & 3.0 & & 4637 & 5.2 & 3007 & $2 \cdot 4$ & 1634 & $1 \cdot 8$ & 1402 & $2 \cdot 2$ & 2534 & $2 \cdot 4$ & & 2902 & 2.5 & 1681 & 2.9 & 3563 & 4.8 \\
\hline $\begin{array}{l}\text { PA (MET-min/week) } \\
\text { Median }\end{array}$ & & 180 & & & 1935 & & 1773 & & $17 \varepsilon$ & 87 & & 773 & & & & 1739 & & 1828 & & 203 & 31 \\
\hline $\begin{array}{l}\text { Q1, Q3 } \\
\text { Percente }\end{array}$ & & 845,3 & 3546 & & 813,415 & & 838,34 & & 874,3 & 3386 & & 3384 & & 334,3345 & & $824,334 \varepsilon$ & & 852,35 & & 897,4 & 4194 \\
\hline $\begin{array}{l}\text { Percentage body fat } \\
\text { Median } \\
\text { Q1, Q3 }\end{array}$ & & $\begin{array}{r}30 \\
24.7\end{array}$ & $\begin{array}{l}2 \\
36.7\end{array}$ & & $\begin{array}{c}29 \cdot 6 \\
24 \cdot 4,36\end{array}$ & & $\begin{array}{r}30.8 \\
24 \cdot 8,3\end{array}$ & & $\begin{array}{r}30 \\
24 \cdot 8\end{array}$ & 34.4 & & 29.6 & & $\begin{array}{c}33 \cdot 9 \\
28 \cdot 0,39 \cdot 2\end{array}$ & & $\begin{array}{r}31 \cdot 2 \\
25 \cdot 0,37 \cdot 6\end{array}$ & & $\begin{array}{r}27 \cdot 7 \\
23 \cdot 1,33\end{array}$ & & $\begin{array}{r}26 . \\
23 \cdot 0\end{array}$ & 31.2 \\
\hline & & & & & & Coffee intal & ake in cups/d & & & & & & & & & Tea intake & ke in cups/d & & & & \\
\hline & & 66840 & & $0.5-1 n$ & $n 98765$ & $2 n 7$ & 71654 & $3-4 n$ & 78677 & $>4 \cap 3$ & 38450 & $0 \cap 48$ & 8959 & $0.5-1$ & $n 42313$ & $2 n 5$ & 53093 & $3-4 n 1$ & 107229 & $>4 n 1$ & 102792 \\
\hline Parameter & Median & Q1, & Q3 & Median & Q1, Q3 & Median & Q1, Q3 & Median & Q1, Q3 & Median & Q1, Q3 & Median & Q1, Q3 & Median & Q1, Q3 & Median & Q1, Q3 & Median & Q1, Q3 & Median & Q1, Q3 \\
\hline $\begin{array}{l}\text { Wine intake (g } \\
\text { alcohol/d) }\end{array}$ & $4 \cdot 3$ & 0.0 & 10.0 & 5.7 & $1 \cdot 6,11 \cdot 4$ & 7.1 & $2 \cdot 9,12 \cdot 9$ & 7.1 & $2 \cdot 9,12 \cdot 9$ & 5.7 & $1 \cdot 3,12 \cdot 9$ & 5.7 & $1 \cdot 0,12 \cdot 9$ & 7.1 & $2 \cdot 9,14 \cdot 3$ & 7.1 & $2 \cdot 9,12 \cdot 9$ & $5 \cdot 7$ & $2 \cdot 9,11 \cdot 4$ & 5.7 & $1.3,10 \cdot 0$ \\
\hline $\begin{array}{l}\text { Non-wine intake (g } \\
\text { alcohol/d) }\end{array}$ & 4.3 & 0.0 & $14 \cdot 3$ & 2.9 & $0.0,11 \cdot 4$ & 4.3 & $0.0,11 \cdot 4$ & 4.3 & $0 \cdot 0,12 \cdot 9$ & 5.7 & $1 \cdot 0,17 \cdot 1$ & $5 \cdot 3$ & $0 \cdot 0,14 \cdot 3$ & 4.3 & $0.0,12 \cdot 9$ & 4.3 & $0.0,11 \cdot 4$ & 4.3 & $0.0,11 \cdot 4$ & 4.3 & $0 \cdot 0,14 \cdot 3$ \\
\hline $\begin{array}{l}\text { Coffee intake } \\
\text { (cups/d) }\end{array}$ & 0.0 & 0.0 & & 1.0 & $0.5,1.0$ & 2.0 & $2 \cdot 0,2 \cdot 0$ & 3.0 & $3 \cdot 0,4 \cdot 0$ & 6.0 & $5 \cdot 0,6 \cdot 0$ & 3.0 & $2 \cdot 0,5 \cdot 0$ & 3.0 & $1 \cdot 0,4 \cdot 0$ & 2.0 & $1 \cdot 0,3 \cdot 0$ & 1.0 & $0.5,3.0$ & 1.0 & $0.0,2.0$ \\
\hline $\begin{array}{l}\text { Tea intake (cups/d) } \\
\text { Age (years) }\end{array}$ & $\begin{array}{c}4.0 \\
56\end{array}$ & $\begin{array}{l}3.0, \\
49,\end{array}$ & & $\begin{array}{l}4 \cdot 0 \\
58\end{array}$ & $\begin{array}{l}2 \cdot 0,5 \cdot 0 \\
51,63\end{array}$ & $\begin{array}{l}3 \cdot 0 \\
59\end{array}$ & $\begin{array}{l}2 \cdot 0,4 \cdot 0 \\
51,64\end{array}$ & $\begin{array}{l}2 \cdot 0 \\
58\end{array}$ & $\begin{array}{l}0.5,4 \cdot 0 \\
51,63\end{array}$ & $\begin{array}{l}1 \cdot 0 \\
56\end{array}$ & $\begin{array}{l}0 \cdot 0,3 \cdot 0 \\
49,62\end{array}$ & $\begin{array}{l}0.0 \\
56\end{array}$ & $\begin{array}{l}0.0,0.0 \\
49,62\end{array}$ & $\begin{array}{l}1.0 \\
56\end{array}$ & $\begin{array}{l}0.5,1 \cdot 0 \\
49,63\end{array}$ & $\begin{array}{l}2 \cdot 0 \\
58\end{array}$ & $\begin{array}{l}2 \cdot 0,2 \cdot 0 \\
50,63\end{array}$ & $\begin{array}{l}3.0 \\
58\end{array}$ & $\begin{array}{l}3 \cdot 0,4 \cdot 0 \\
51,63\end{array}$ & $\begin{array}{l}6 \cdot 0 \\
58\end{array}$ & $\begin{array}{l}5 \cdot 0,7 \cdot 0 \\
51,63\end{array}$ \\
\hline & & $\%$ & & & $\%$ & & $\%$ & & $\%$ & $n$ & $\%$ & & $\%$ & $n$ & $\%$ & & $\%$ & $n$ & $\%$ & & $\%$ \\
\hline $\begin{array}{l}\text { Female } \\
\text { Smoking status }\end{array}$ & 35568 & $53 \cdot 2$ & & 52565 & $53 \cdot 2$ & 36786 & $51 \cdot 3$ & 37946 & $48 \cdot 2$ & 16811 & 43.7 & 25585 & $52 \cdot 3$ & 20603 & 48.7 & 26401 & 49.7 & 55711 & 52.0 & 51376 & 50.0 \\
\hline & 36708 & 54.9 & & 55019 & $55 \cdot 7$ & 38650 & 53.9 & 40452 & 51.4 & 16538 & 43.0 & 24636 & $50 \cdot 3$ & 22005 & $52 \cdot 0$ & 28446 & 53.6 & 58668 & 54.7 & 53612 & $52 \cdot 2$ \\
\hline & 23771 & 35.6 & & 36493 & $36 \cdot 9$ & 26954 & 37.6 & 30004 & 38.1 & 14635 & & 17989 & $36 \cdot 7$ & 15757 & 37.2 & 20038 & $37 \cdot 7$ & 40046 & $37 \cdot 3$ & 38027 & 37.0 \\
\hline Current & 6361 & 9.5 & & 7253 & $7 \cdot 3$ & 6050 & 8.4 & 8221 & $10 \cdot 4$ & 7277 & 18.9 & 6334 & $12 \cdot 9$ & 4551 & 10.8 & 4609 & 8.7 & 8515 & 7.9 & 11153 & $10 \cdot 9$ \\
\hline AHI (k£) & & & & & & & & & & & & & & & & & & & & & \\
\hline & 12396 & 18.5 & & 16161 & 16.4 & 10764 & $15 \cdot 0$ & 11044 & 14.0 & 6137 & $16 \cdot 0$ & 7932 & $16 \cdot 2$ & 5584 & 13.2 & 7513 & $14 \cdot 2$ & 17013 & $15 \cdot 9$ & 18460 & $18 \cdot 0$ \\
\hline & & 21 & & & 22 & & & & & & & & & & & & & & & & $22 \cdot 3$ \\
\hline $31-<52$ & 155 & $23 \cdot 3$ & & & 23.6 & 123 & $24 \cdot 2$ & 196 & 25.0 & & 24.5 & 11941 & 24. & 10536 & 24.9 & 128 & $24 \cdot 3$ & 25735 & 24.0 & 24387 & $23 \cdot 7$ \\
\hline $52-<100$ & 12611 & 18.9 & & 19564 & 19.8 & 14642 & $20 \cdot 4$ & 16826 & $21 \cdot 4$ & 8264 & 21.5 & 9931 & $20 \cdot 3$ & 9766 & $23 \cdot 1$ & 11625 & 21.9 & 21194 & 19.8 & 19391 & 18.9 \\
\hline$\geq 100$ & 3004 & 4.5 & & 5612 & 5.7 & 4402 & $6 \cdot 1$ & 5006 & 6.4 & 2148 & $5 \cdot 6$ & 2680 & 5.5 & 3340 & 7.9 & 3579 & 6.7 & 5949 & $5 \cdot 5$ & 4624 & 4.5 \\
\hline
\end{tabular}


population was $58(50,63)$ with $50 \cdot 7 \%$ of participants being female. Median intake was $5.7(1.4,11.4) \mathrm{g}$ alcohol/d from wine, $4.3(0 \cdot 0,12 \cdot 9) \mathrm{g}$ alcohol/d from non-wine, $2 \cdot 0(0 \cdot 5,3 \cdot 0) \mathrm{cups} / \mathrm{d}$ coffee and $3 \cdot 0(1 \cdot 0,5 \cdot 0) \mathrm{cups} / \mathrm{d}$ tea (Table 1$)$. Median (Q1, Q3) wine intake was similar in female $(5.7(2.9,11.4) \mathrm{g}$ alcohol/d) compared with male $(5 \cdot 7(0.7,11.4) \mathrm{g}$ alcohol/d) participants (online Supplementary Table 1). Non-wine intake was lower in females $(1.4(0 \cdot 0,4 \cdot 3) \mathrm{g}$ alcohol/d) as compared with males (11.4 (4.3, 22.9) g alcohol/d) (online Supplementary Table 1). Furthermore, median coffee (females $2 \cdot 0(0 \cdot 5,3 \cdot 0)$; males $2 \cdot 0$ $(1 \cdot 0,3 \cdot 0)$ cups/d) and tea (females and males $3 \cdot 0(1 \cdot 0,5 \cdot 0))$ intake was similar between both sexes (online Supplementary Table 1$)$. Follow-up was $12 \cdot 0(11 \cdot 3,12 \cdot 7)$ years with $4 \cdot 2$ million person-years.

\section{Beverage intake and all-cause mortality}

Overall, 7243 deaths occurred in females and 12958 in males, that is, a total of 20201 deaths.

Wine intake. In all participants, a significant U-shaped association between wine intake and all-cause mortality was detected with the nadir at $20 \mathrm{~g}$ alcohol/d (Fig. 1(a)). Similar findings were observed in sex-dependent analyses with the nadir for all-cause mortality at $20 \mathrm{~g}$ alcohol/d for both females (Fig. 1(b)) and males (Fig. 1(c)). HR ${ }^{0}$ was $1.07(1.03,1.12)$ in females and 1.14 (1.11, 1.17) in males (Fig. 1(b)-(c)). A significant U-shaped association between wine intake and all-cause mortality was also detected in sensitivity analyses in cohorts S1 and S2 with the nadir between 19 and $20 \mathrm{~g}$ alcohol/d from wine in all participants, as well as in females and males separately (online Supplementary Fig. 2). In cohort $\mathrm{S} 2, \mathrm{HR}^{0}$ for present non-alcohol drinkers was higher in both females $(1.23(1.20,1.26)$; online Supplementary Fig. 2(d)) and males (1.20 (1.18, 1.22); Supplementary Fig. 2(f)) compared with the corresponding $\mathrm{HR}^{0}$ in the primary cohort (Fig. 1(b)-(c)).

Non-wine intake. In all participants and in females, a significant positive dose-dependent association between non-wine intake and all-cause mortality was detected with the nadir at $0 \mathrm{~g}$ alcohol/d (Fig. 2(a)-(b)). In males, the nadir was at $9 \mathrm{~g}$ alcohol/d from non-wine with dose-dependent increases seen beyond $20 \mathrm{~g}$ alcohol/d (Fig. 2(c)). The shape of the association between non-wine intake and all-cause mortality changed towards a J-shaped curve in the sensitivity analyses with the most pronounced alterations seen in cohort S2 (online Supplementary Fig. 3). In cohort S2, the nadir was at 14,7 and $17 \mathrm{~g}$ alcohol/d in all participants, females and males, respectively (online Supplementary Fig. 3(b), (d) and (f)).

Coffee intake. In all participants, coffee intake and all-cause mortality were significantly associated (Fig. 3(a)). The nadir was observed at 2 cups/d coffee and $\mathrm{HR}^{0}$ was significantly increased at $1.06(1.03,1.09)$ (Fig. 3(a)). Association was similar in females and males; however, statistical significance was not reached in both sexes (Fig. 3(b) and (c)). Findings were similar in sensitivity analyses using cohorts S1 and S2 (data not shown). 
Tea intake. In all participants, all-cause mortality risk continuously decreased from $\mathrm{HR}^{0}$ of $1 \cdot 18(1 \cdot 14,1 \cdot 21)$ to the nadir at 4 cups/d and no significant effects at higher consumption levels (Fig. 4(a)). Similar to all participants, $\mathrm{HR}^{0}$ was significantly increased in both females $(1 \cdot 19(1 \cdot 13,1 \cdot 25))$ and males $(1 \cdot 17$ $(1 \cdot 12,1 \cdot 21))$ compared with the HR at the nadir (females: 7 , males: 4 cups/d), respectively (Fig. 4(b) and (c)). Almost identical results were obtained in sensitivity analyses in cohorts $\mathrm{S} 1$ and S2 (data not shown).

\section{Beverage intake and cancer mortality}

Within all-cause mortality, 4520 cancer deaths occurred in females and 6360 in males, that is, a total of 10880 cancer deaths. All findings concerning the association between beverage intake and cancer mortality are summarised in online Supplementary Table 2.

Wine intake. In all participants and in both sexes separately, wine intake was not significantly associated with cancer mortality. Findings were numerically similar in cohorts S1 and S2, with associations reaching statistical significance in the total and male S2 cohort.

Non-wine intake. In all participants, as well as in females and males separately, non-wine intake was significantly associated with cancer mortality in a linear way. The nadir was at 0,0 and $3 \mathrm{~g}$ alcohol/d from non-wine in all participants, females and males, respectively. Significant linear associations were also observed in cohorts S1 and S2. However, the nadir was seen at higher intake levels with a most pronounced shift towards higher values in cohort $\mathrm{S} 2$.

Coffee intake. Coffee intake was not significantly associated with cancer mortality in all participants, females and males in the primary cohort, as well as in cohorts S1 and S2.

Tea intake. Tea consumption was significantly associated in a non-linear form with cancer mortality and $\mathrm{HR}^{0}$ was significantly increased in all participants and both sexes separately. The nadir was between 3 (females) and 4 (all participants, males) cups/d. Almost identical findings were observed in cohorts S1 and S2.

\section{Beverage intake and non-cancer mortality}

Within all-cause mortality, 2723 non-cancer deaths occurred in females and 6598 in males, that is, a total of 9321 non-cancer deaths. All findings concerning the association between beverage intake and non-cancer mortality are summarised in online Supplementary Table 3 .

Wine intake. Wine consumption was significantly associated with non-cancer mortality in a U-shaped form in all participants and both sexes separately. The nadir was between 21 (all participants, females) and 23 (males) $\mathrm{g}$ alcohol/d from wine. $\mathrm{HR}^{0}$ was significantly increased at $1 \cdot 21(1 \cdot 18,1 \cdot 25)$ in all participants, 1.25 $(1 \cdot 17,1 \cdot 33)$ in females and $1.19(1 \cdot 15,1.23)$ in males. Whereas the nadir was similar in cohorts $\mathrm{S} 1$ and $\mathrm{S} 2, \mathrm{HR}^{0}$ was numerically higher with most pronounced increases seen in cohort S2.
Non-wine intake. In all participants and in females, a significant positive dose-dependent association between non-wine intake and non-cancer mortality was detected with the nadir at $0 \mathrm{~g}$ alco$\mathrm{hol} / \mathrm{d}$. In males, HR was lowest between 0 and 20 (nadir at 12) $\mathrm{g}$ alcohol/d from non-wine with dose-dependent increases seen beyond $20 \mathrm{~g}$ alcohol/d. The shape of the association between non-wine intake and non-cancer mortality changed towards a J-shaped curve in the sensitivity analyses with the most pronounced alterations seen in cohort S2. Here, the nadir was at 17,8 and $19 \mathrm{~g}$ alcohol/d and $\mathrm{HR}^{0}$ was significantly elevated at $1 \cdot 11(1 \cdot 09,1 \cdot 13), 1 \cdot 04(1 \cdot 02,1 \cdot 07)$ and $1 \cdot 14(1 \cdot 11,1 \cdot 18)$ in all participants, females and males, respectively.

Coffee intake. Coffee intake was significantly associated with non-cancer mortality in a non-linear manner in all participants and males. The nadir was observed at $2 \mathrm{cups} / \mathrm{d}$ and $\mathrm{HR}^{0}$ was slightly but significantly elevated at $1.08(1.04,1.13)$ and 1.07 $(1 \cdot 02,1 \cdot 12)$. In contrast, no significant association was observed in female subjects. Similar findings were obtained in cohorts S1 and $\mathrm{S} 2$ with $\mathrm{p}^{\text {non-lin }}$ also becoming significant in females.

Tea intake. Tea consumption was significantly associated in a linear and non-linear way with non-cancer mortality and $\mathrm{HR}^{0}$ was significantly elevated in all participants and both sexes separately. The nadir was between 5 (all participants, males) and 9 (females) cups/d. Similar results were observed in cohorts S1 and S2.

\section{Beverage intake and CVD mortality}

Within non-cancer deaths, 916 CVD deaths occurred in females and 2858 in males, that is, a total of 3774 CVD deaths. All findings concerning the association between beverage intake and CVD mortality are summarised in online Supplementary Table 4.

Wine intake. Wine intake was significantly associated with CVD mortality in a non-linear manner in all participants and both sexes separately. The nadir was between 19 (females) and 21 (males) $\mathrm{g}$ alcohol/d. $\mathrm{HR}^{0}$ was significantly increased at 1.22 $(1 \cdot 16,1.28)$ in all participants, $1.31(1.17,1.46)$ in females and $1.20(1.14,1.26)$ in males. Whereas the nadir was similar in cohorts $\mathrm{S} 1$ and $\mathrm{S} 2, \mathrm{HR}^{0}$ was numerically higher with the most pronounced increases seen in cohort S2.

Non-wine intake. In all participants and in males, a significant positive dose-dependent association between non-wine intake and CVD mortality was detected with the nadir at 4 and $6 \mathrm{~g}$ alco$\mathrm{hol} / \mathrm{d}$, respectively. The shape of the association between nonwine intake and CVD mortality changed towards a J-shaped curve in the sensitivity analyses with the most pronounced alterations seen in cohort S2. Here, the nadir was at 16 and $17 \mathrm{~g}$ alco$\mathrm{hol} / \mathrm{d}$ and $\mathrm{HR}^{0}$ was significantly elevated at $1.08(1 \cdot 05,1 \cdot 11)$ and $1.08(1.03,1.13)$ in all participants and males, respectively. In contrast, no significant association was observed in females in the primary cohort, as well as in cohorts S1 and S2. 
Coffee intake. Coffee intake was not significantly associated with CVD mortality in all participants, females and males in the primary cohort, as well as in cohorts S1 and S2.

Tea intake. Tea consumption was significantly associated in a non-linear way in all participants and males, as well as in a linear negative manner in females. $\mathrm{HR}^{0}$ was significantly increased at $1.20(1 \cdot 12,1 \cdot 29), 1 \cdot 55(1.34,1.78)$ and $1.18(1.09,1 \cdot 28)$ in all participants, females and males, respectively, with the nadir ranging from 4 (males) to 10 (females) cups/d. Similar findings were obtained in cohorts S1 and S2.

\section{Discussion}

In the present study, it is elucidated for the first time how wine, non-wine, coffee and tea intake included as continuous non-linear predictors and mutually adjusted are associated with allcause and cause-specific mortality.

For all participants and in sex-dependent analyses (primary cohort), a significant U-shaped association is seen between wine intake and all-cause mortality with $\mathrm{HR}^{0}$ significantly increased as compared with the nadir at $20 \mathrm{~g}$ alcohol/d. A decreased risk of death for light to moderate wine intake has also been shown in studies from the USA ${ }^{(45)}$, Denmark ${ }^{(46)}$, France ${ }^{(47)}$ and Sweden ${ }^{(48)}$ comprising 128 934, 24 523, 36250 and 1828 individuals, respectively. To the best of our knowledge, only one study so far has assessed the sex-dependent association between different intake levels of wine and all-cause mortality. In the study by Baglietto and co-workers, HR of death is lowest in the categories 1-19 and 20-39 $\mathrm{g}$ alcohol/d from wine in females and males, respectively ${ }^{(49)}$. Similar to the current findings but using a different analytical approach, Jani and co-workers demonstrate convincingly in UK Biobank participants that HR for death decreases in red wine consumers up to around 20 weekly alcohol units ${ }^{(13)}$. Furthermore, red wine and champagne plus white wine intake is inversely related to all-cause mortality in another study based on UK Biobank participants in categorical analyses; however, this association largely disappears in a continuous analysis ${ }^{(29)}$. A different analytical approach and follow-up interval might well explain the differences between the latter results and our current findings. The present study is the first to elucidate the association between wine intake and non-cancer mortality. We show a significant Ushaped association in all participants and both sexes separately with increased $\mathrm{HR}^{0}$ as compared with the nadir observed between 21 and $23 \mathrm{~g}$ alcohol/d from wine. Similar results are obtained for CVD with the nadir detected between 19 and $21 \mathrm{~g}$ alcohol/d from wine. Our CVD results are well in accordance with a meta-analysis comprising six cohort studies, and the lowest risk of death was found at $24 \mathrm{~g}$ alcohol $/ \mathrm{d}$ from wine $\mathrm{e}^{(30)}$.

To the best of our knowledge, no study so far has contrasted non-wine with wine consumption regarding mortality after excluding non-drinkers. In contrast to wine consumption, a positive dose-dependent relation exists in the primary cohort between non-wine intake and all-cause mortality with the nadir observed at 0,0 and $9 \mathrm{~g}$ alcohol/d from non-wine in all participants, females and males, respectively. Within the non-wine category, several studies have assessed the association of beer and spirits with all-cause mortality. In agreement with our nonwine results, Schutte and co-workers demonstrate convincingly that both beer/cider and spirits intake is associated with increased all-cause mortality risk in UK Biobank participants ${ }^{(29)}$. Similarly, beer/cider and spirits drinkers have a significantly higher all-cause mortality risk if compared with red wine drinkers ${ }^{(13)}$. A lower mortality risk in drinkers of any type of wine as compared with beer or spirits drinkers is also found in an independent cohort ${ }^{(45)}$. In another report, HR for all-cause mortality is increased in the highest category of beer and spirits consumption in males but not females ${ }^{(49)}$. In agreement with our findings, non-wine consumption is positively associated with cancer mortality in a cohort from Denmark $^{(46)}$. Interestingly, beer/cider and spirits consumption is also associated with an increased risk for incident cancer in UK Biobank participants ${ }^{(29)}$. To the best of our knowledge, our study is the first to show a significant positive dose-dependent association between non-wine intake and non-cancer mortality. Within non-cancer, non-wine intake is positively and dose-dependently related to CVD mortality. Interestingly, beer/cider and spirits consumption is associated with an increased risk for cardiovascular events, ischaemic heart disease and cerebrovascular disease in UK Biobank participants ${ }^{(29)}$. In contrast to the current findings, a J-shaped relationship with CVD mortality is apparent for beer but not spirits ${ }^{(30)}$. However, this meta-analysis has not consistently controlled for the impact of non-drinkers on mortality ${ }^{(30)}$. Combined these findings suggest that associations between wine and non-wine consumption on the one hand and different types of mortality on the other hand show opposite directions in many cases at low to moderate drinking levels causing underestimation of mortality risk when pooled into one alcohol variable.

To the best of our knowledge, no study so far has defined the impact of not controlling for never or former alcohol drinking on the association between wine or non-wine intake and mortality. In the current study, $\mathrm{HR}^{0}$ for wine or non-wine increases further if never drinkers (cohort S1) and all non-alcohol drinkers (cohort S2) are included in the analysis with most pronounced changes observed in cohort S2. Decreased mortality in low to moderate alcohol drinkers in cohorts S1 and S2 could be explained by a higher death risk of former and never drinkers who might have quit or not initiated alcohol consumption because of poor health ${ }^{(33,34)}$. These findings support the notion that the handling of non-drinkers has a major impact on mortality analyses and might explain some discrepancies between studies. Thus, two reports demonstrate convincingly that light to moderate wine consumption is significantly related to a lower risk of death from cancer $^{(46,47)}$ in contrast to our present results in the primary cohort. However, if we perform our analysis in cohort S2 similar to the approach used in both studies ${ }^{(46,47)}$, we also observe a significant non-linear association between wine intake and an increased $\mathrm{HR}^{0}$ for cancer deaths. Furthermore, non-wine intake of up to 21 drinks/week is not associated with an increased risk of death in one study ${ }^{(46)}$ in contrast to the current findings in the primary cohort. However, if we perform our analysis in cohort S2 similar to the approach used in this study ${ }^{(46)}$, the nadir increases to $14 \mathrm{~g}$ alcohol/d and risk of death is not increased up to about $25 \mathrm{~g}$ alco$\mathrm{hol} / \mathrm{d}$ from non-wine as compared with $\mathrm{HR}^{0}$. 
For coffee intake, the nadir is observed at 2 cups/d with nonsignificant effects at higher consumption levels and slightly but significantly increased $\mathrm{HR}^{0}$ in all participants. In agreement with the present results, increased all-cause mortality risk in non-coffee drinkers is shown in previous studies ${ }^{(26-28,50)}$. However, the nadir varies and is somewhat higher compared with the present report, that is, between 3 and 7 cups/ $\mathrm{d}^{(26-28,50-53)}$. Loftfield and co-workers ${ }^{(53)}$ also assess UK Biobank participants with differences in study results being well explained by different exclusion criteria, follow-up time and model adjustments with coffee intake included as an ordinal variable. Coffee intake is not significantly associated with cancer mortality in the present analysis in agreement with most studies ${ }^{(26,54-56)}$ except one ${ }^{(27)}$. In the current study, the nadir for non-cancer mortality is $2 \mathrm{cups} / \mathrm{d}$ coffee in all participants with no further effects at higher amounts and a significantly increased $\mathrm{HR}^{0}$. To the best of our knowledge, no study so far has assessed the relation between coffee intake and overall non-cancer mortality. Within noncancer, no significant association is detected between coffee consumption and CVD mortality. However, a dose-dependent decrease in CVD mortality as suggested by previous meta-analyses ${ }^{(26,27)}$ cannot be ruled out since coffee consumption levels show a nadir numerically between 2 (male) and 8 (female) cups/d. Taking published and current findings into consideration, coffee is not positively related to all-cause, cancer, noncancer and CVD mortality. A minor negative dose-dependent association remains possible.

Tea consumption shows a significant negative dose-dependent association with all-cause mortality, and $\mathrm{HR}^{0}$ is significantly increased compared with HR at the nadir. Results are similar in both sexes. The nadir for all-cause mortality is between 4 and 7 cups/d tea similar to published results where it is found between 2 and $>5 \mathrm{cups} / \mathrm{d}^{(20,24,57,58)}$. Similar to the present findings for allcause mortality, tea consumption is associated with decreased cancer mortality. Although the type of tea has not been recorded during baseline assessment, the majority of tea drinkers probably consumes black tea since Great Britain has one of the highest per capita black tea consumption worldwide ${ }^{(59)}$. It is interesting to note in this context that the intake of black but not green tea has been linked with lower cancer mortality ${ }^{(20)}$. To the best of our knowledge, the present study is the first to show that $\mathrm{HR}^{0}$ for non-cancer mortality compared with HR at the nadir (5-9 cups/d) is significantly increased in both sexes. Findings are similar for CVD in agreement with previous reports observing a negative dose-dependent association between tea and CVD mortality in both sexes ${ }^{(24,57,60)}$. Taking previous publications and the present findings into consideration, tea consumption is consistently and significantly associated with decreased allcause, cancer, non-cancer and CVD mortality in both sexes with the nadir ranging from 3 to $10 \mathrm{cups} / \mathrm{d}$ and no increases in risk of death at higher doses.

Strengths of the current report include the prospective cohort design, a large sample size, thorough characterisation of UK Biobank participants, median follow-up time $>10$ years, as well as the wide range of wine, non-wine, coffee and tea intake included as continuous parameters. Limitations include that consumption of other important beverages such as sugar-sweetened beverages and milk-based drinks, as well as type of tea, has not been assessed during the baseline visit. Furthermore, the present results cannot be adjusted for energy intake since this parameter was not assessed during the UK Biobank baseline visit. Further potential limitations include residual confounding, as well as measurement errors in the assessment of the exposure variables, potential confounders and misclassification of cause of death. Two studies have evaluated the performance of the UK Biobank touchscreen dietary questionnaire. As shown convincingly by Bradbury et $a l^{(61)}$ and Carter et al. ${ }^{(62)}$, the touchscreen questionnaire adequately discriminates between high and low intakes for selected food groups when compared with a 24-h dietary assessment (Oxford WebQ). The level of agreement is comparable with estimates reported between traditional 24-h recalls and food frequency questionnaires in previous studies ${ }^{(61,62)}$. In addition, causal mediation analysis for specific covariates, for example, percentage body fat, could not be performed since it has not been implemented in R for Cox proportional hazard regression models with covariates included as penalised cubic splines. Moreover, a 'healthy volunteer' selection bias might exist since the cohort is not demographically representative of the general UK population ${ }^{(63)}$. However, a representative population is not required to define exposure-disease relationships ${ }^{(63)}$.

Summarising on a population level, the current study indicates that light to moderate consumption of wine but not nonwine is associated with decreased all-cause and non-cancer mortality. Coffee consumption is not related to increased mortality and a minor negative dose-dependent association remains possible. Tea intake is associated with a consistently decreased risk of all mortality types studied in both sexes. Further prospective studies on beverage intake in relation to morbidity from cancer and non-cancer disease are necessary to provide even more definitive conclusions.

\section{Acknowledgements}

This research has been conducted using the UK Biobank Resource, application number 53438.

This work was supported by the Deutsche Forschungsgemeinschaft (DFG) (SFB 1052/2 C6) and the Deutsche Diabetes Stiftung to MF. The funders had no role in the design, analysis or writing of this article.

S. M. S. and A. K. conceived the research. S. M. S., A. K. and M. F. wrote the paper. Statistical analyses were performed by all authors. All authors have read, redacted and approved the final manuscript.

There are no conflicts of interest.

\section{Supplementary material}

For supplementary material/s referred to in this article, please visit https://doi.org/10.1017/S000711452200040X 


\section{References}

1. Bechthold A, Boeing H, Schwedhelm C, et al. (2019) Food groups and risk of coronary heart disease, stroke and heart failure: a systematic review and dose-response meta-analysis of prospective studies. Crit Rev Food Sci Nutr 59, 1071-1090.

2. Schwingshackl L, Schwedhelm C, Hoffmann G, et al. (2017) Food groups and risk of all-cause mortality: a systematic review and meta-analysis of prospective studies. Am J Clin Nutr 105, 1462-1473.

3. Qin P, Li Q, Zhao Y, et al. (2020) Sugar and artificially sweetened beverages and risk of obesity, type 2 diabetes mellitus, hypertension, and all-cause mortality: a dose-response metaanalysis of prospective cohort studies. Eur J Epidemiol 35, 655-671.

4. Chazelas E, Srour B, Desmetz E, et al. (2019) Sugary drink consumption and risk of cancer: results from NutriNet-Santé prospective cohort. BMJ 366, 12408.

5. Department of Health (2016) UK Chief Medical Officers' Alcohol Guidelines Review: Summary of the Proposed New Guidelines. https://assets.publishing.service.gov.uk/government/uploads/ system/uploads/attachment_data/file/489795/summary.pdf (accessed November 2021).

6. Li X-H, Yu F-F, Zhou Y-H, et al. (2016) Association between alcohol consumption and the risk of incident type 2 diabetes: a systematic review and dose-response meta-analysis. Am J Clin Nutr 103, 818-829.

7. Mostofsky E, Chahal HS, Mukamal KJ, et al. (2016) Alcohol and immediate risk of cardiovascular events: a systematic review and dose-response meta-analysis. Circulation 133, 979-987.

8. Roerecke M, Tobe SW, Kaczorowski J, et al. (2018) Sex-specific associations between alcohol consumption and incidence of hypertension: a systematic review and meta-analysis of cohort studies. J Am Heart Assoc 7, e008202.

9. Roerecke M, Vafaei A, Hasan OSM, et al. (2019) Alcohol consumption and risk of liver cirrhosis: a systematic review and meta-analysis. Am J Gastroenterol 114, 1574-1586.

10. Choi Y-J, Myung S-K \& Lee J-H (2018) Light alcohol drinking and risk of cancer: a meta-analysis of cohort studies. Cancer Res Treat 50, 474-487.

11. Huang J, Wang X \& Zhang Y (2017) Specific types of alcoholic beverage consumption and risk of type 2 diabetes: a systematic review and meta-analysis. J Diabetes Investig 8, 56-68.

12. Inan-Eroglu E, Powell L, Hamer M, et al. (2020) Is there a link between different types of alcoholic drinks and obesity? An analysis of 280,183 UK Biobank participants. Int J Environ Res Public Health 17, 14.

13. Jani BD, McQueenie R, Nicholl BI, et al. (2021) Association between patterns of alcohol consumption (beverage type, frequency and consumption with food) and risk of adverse health outcomes: a prospective cohort study. BMC Med 19, 8 .

14. ODPHP (2015) Dietary Guidelines for Americans. https:// health.gov/sites/default/files/2019-09/2015-2020_Dietary_ Guidelines.pdf (accessed November 2021).

15. Ding M, Bhupathiraju SN, Chen M, et al. (2014) Caffeinated and decaffeinated coffee consumption and risk of type 2 diabetes: a systematic review and a dose-response meta-analysis. Diabetes Care 37, 569-586.

16. Lee A, Lim W, Kim S, et al. (2019) Coffee intake and obesity: a meta-analysis. Nutrients 11, 1274

17. Ding M, Bhupathiraju SN, Satija A, et al. (2014) Long-term coffee consumption and risk of cardiovascular disease: a systematic review and a dose-response meta-analysis of prospective cohort studies. Circulation 129, 643-659.

18. Zhao L-G, Li Z-Y, Feng G-S, et al. (2020) Coffee drinking and cancer risk: an umbrella review of meta-analyses of observational studies. BMC Cancer 20, 101.
19. EFSA Panel on Dietetic Products, Nutrition and Allergies (2015) Scientific opinion on the safety of caffeine. EFSA J 13, 4102.

20. Yi M, Wu X, Zhuang W, et al. (2019) Tea consumption and health outcomes: umbrella review of meta-analyses of observational studies in humans. Mol Nutr Food Res $\mathbf{6 3}$, e1900389.

21. Li X, Yu C, Guo Y, et al. (2017) Tea consumption and risk of ischaemic heart disease. Heart 103, 783-789.

22. Kim TL, Jeong GH, Yang JW, et al. (2020) Tea consumption and risk of cancer: an umbrella review and meta-analysis of observational studies. Adv Nutr 11, 1437-1452.

23. Bagnardi V, Rota M, Botteri E, et al. (2013) Light alcohol drinking and cancer: a meta-analysis. Ann Oncol 24, 301-308.

24. Tang J, Zheng J-S, Fang L, et al. (2015) Tea consumption and mortality of all cancers, CVD and all causes: a meta-analysis of eighteen prospective cohort studies. Br J Nutr 114, 673-683.

25. Di Castelnuovo A, Costanzo S, Bagnardi V, et al. (2006) Alcohol dosing and total mortality in men and women: an updated meta-analysis of 34 prospective studies. Arch Intern Med 166, 2437-2445.

26. Crippa A, Discacciati A, Larsson SC, et al. (2014) Coffee consumption and mortality from all causes, cardiovascular disease, and cancer: a dose-response meta-analysis. Am J Epidemiol 180, 763-775.

27. Kim Y, Je Y \& Giovannucci E (2019) Coffee consumption and all-cause and cause-specific mortality: a meta-analysis by potential modifiers. Eur J Epidemiol 34, 731-752.

28. Poole R, Kennedy OJ, Roderick P, et al. (2017) Coffee consumption and health: umbrella review of meta-analyses of multiple health outcomes. BMJ 359, j5024.

29. Schutte R, Papageorgiou M, Najlah M, et al. (2020) Drink types unmask the health risks associated with alcohol intake prospective evidence from the general population. Clin Nutr 39, 3168-3174

30. Costanzo S, Di Castelnuovo A, Donati MB, et al. (2011) Wine, beer or spirit drinking in relation to fatal and non-fatal cardiovascular events: a meta-analysis. Eur J Epidemiol 26, 833-850.

31. UK Biobank (2007) UK Biobank: Protocol for a Large-Scale Prospective Epidemiological Resource. https://www. ukbiobank.ac.uk/media/gnkeyh2q/study-rationale.pdf (accessed November 2021).

32. Behrendt I, Fasshauer M \& Eichner G (2021) Gluten intake and metabolic health: conflicting findings from the UK Biobank. Eur J Nutr 60, 1547-1559.

33. Stockwell T, Zhao J, Panwar S, et al. (2016) Do "moderate" drinkers have reduced mortality risk? A systematic review and meta-analysis of alcohol consumption and all-cause mortality. J Stud Alcohol Drugs 77, 185-198.

34. Naimi TS, Stockwell T, Zhao J, et al. (2017) Selection biases in observational studies affect associations between 'moderate' alcohol consumption and mortality. Addiction 112, 207-214.

35. Bradbury KE, Murphy N \& Key TJ (2020) Diet and colorectal cancer in UK Biobank: a prospective study. Int J Epidemiol 49, 246-258.

36. UK Biobank (2020) Mortality Data: Linkage to Death Registries. Version 2.0. https://biobank.ctsu.ox.ac.uk/crystal/crystal/ docs/DeathLinkage.pdf (accessed October 2021).

37. R Core Team (2021) R: A Language and Environment for Statistical Computing. https://www.r-project.org (accessed October 2021).

38. Wickham H \& Bryan J (2019) Readxl: Read Excel Files. R Package Version 1.3.1. https://CRAN.R-project.org/package $=$ readxl (accessed October 2021). 
39. Wickham H (2021) Tidyverse: Easily Install and Load the 'Tidyverse'. R Package Version 1.3.1. https://CRAN.R-project. org/package=tidyverse (accessed October 2021).

40. Dusa A (2021) Venn: Draw Venn Diagrams. R Package Version 1.10. https://CRAN.R-project.org/package=venn (accessed October 2021).

41. Quinn M, McNamara A, de la Rubia EA, et al. (2021) Skimr: Compact and Flexible Summaries of Data. R Package Version 2.1.3. https://CRAN.R-project.org/package $=$ skimr (accessed October 2021).

42. Therneau TM (2021) A Package for Survival Analysis in S. Version 3.2-11. https://CRAN.R-project.org/package=survival (accessed October 2021).

43. Therneau TM (2021) Spline Terms in a Cox Model. https://cran. r-project.org/web/packages/survival/vignettes/splines.pdf (accessed November 2021).

44. Therneau TM (2000) Modeling Survival Data. New York, NY: Springer-Verlag.

45. Klatsky AL, Friedman GD, Armstrong MA, et al. (2003) Wine, liquor, beer, and mortality. Am J Epidemiol 158, 585-595.

46. Grønbaek M, Becker U, Johansen D, et al. (2000) Type of alcohol consumed and mortality from all causes, coronary heart disease, and cancer. Ann Intern Med 133, 411-419.

47. Renaud SC, Guéguen R, Siest G, et al. (1999) Wine, beer, and mortality in middle-aged men from eastern France. Arch Intern Med 159, 1865-1870.

48. Theobald H, Bygren LO, Carstensen J, et al. (2000) A moderate intake of wine is associated with reduced total mortality and reduced mortality from cardiovascular disease. J Stud Alcohol 61, 652-656.

49. Baglietto L, English DR, Hopper JL, et al. (2006) Average volume of alcohol consumed, type of beverage, drinking pattern and the risk of death from all causes. Alcohol Alcohol 41, 664-671.

50. Zhao Y, Wu K, Zheng J, et al. (2015) Association of coffee drinking with all-cause mortality: a systematic review and metaanalysis. Public Health Nutr 18, 1282-1291.

51. Ruggiero E, Di Castelnuovo A, Costanzo S, et al. (2021) Daily coffee drinking is associated with lower risks of cardiovascular and total mortality in a general Italian population: results from the Moli-Sani study. J Nutr 151, 395-404.

52. Freedman ND, Park Y, Abnet CC, et al. (2012) Association of coffee drinking with total and cause-specific mortality. N Engl J Med 366, 1891-1904.
53. Loftfield E, Cornelis MC, Caporaso N, et al. (2018) Association of coffee drinking with mortality by genetic variation in caffeine metabolism: findings from the UK Biobank. JAMA Intern Med 178, 1086-1097.

54. Loftfield E, Freedman ND, Graubard BI, et al. (2015) Association of coffee consumption with overall and cause-specific mortality in a large US prospective cohort study. Am J Epidemiol 182, 1010-1022.

55. Ong J-S, Law MH, An J, et al. (2019) Association between coffee consumption and overall risk of being diagnosed with or dying from cancer among 300000 UK Biobank participants in a large-scale Mendelian randomization study. Int J Epidemiol 48, $1447-1456$.

56. Sartini M, Bragazzi NL, Spagnolo AM, et al. (2019) Coffee consumption and risk of colorectal cancer: a systematic review and meta-analysis of prospective studies. Nutrients 11, 3 .

57. Abe SK, Saito E, Sawada N, et al. (2019) Green tea consumption and mortality in Japanese men and women: a pooled analysis of eight population-based cohort studies in Japan. Eur J Epidemiol 34, 917-926.

58. Zhang C, Qin Y-Y, Wei X, et al. (2015) Tea consumption and risk of cardiovascular outcomes and total mortality: a systematic review and meta-analysis of prospective observational studies. Eur J Epidemiol 30, 103-113.

59. Beresniak A, Duru G, Berger G, et al. (2012) Relationships between black tea consumption and key health indicators in the world: an ecological study. BMJ Open 2, e000648.

60. Qiu L, Sautter J \& Gu D (2012) Associations between frequency of tea consumption and health and mortality: evidence from old Chinese. Br J Nutr 108, 1686-1697.

61. Bradbury KE, Young HJ, Guo W, et al. (2018) Dietary assessment in UK Biobank: an evaluation of the performance of the touchscreen dietary questionnaire. J Nutr Sci 7 , e6.

62. Carter JL, Lewington S, Piernas C, et al. (2019) Reproducibility of dietary intakes of macronutrients, specific food groups, and dietary patterns in 211050 adults in the UK Biobank study. J Nutr Sci 8, e34.

63. Fry A, Littlejohns TJ, Sudlow C, et al. (2017) Comparison of sociodemographic and health-related characteristics of UK Biobank participants with those of the general population. Am J Epidemiol 186, 1026-1034. 